\title{
Factors Related to the Probable PTSD after the 9/11 World Trade Center Attack among Asian Americans
}

\author{
Winnie W. Kung • Xinhua Liu • Debbie Huang • \\ Patricia Kim • Xiaoran Wang • Lawrence H. Yang
}

Published online: 15 February 2018

(C) The New York Academy of Medicine 2018

\begin{abstract}
Despite the fact that Asians constituted a sizeable proportion of those exposed to the World Trade Center attack on September 11, 2001 due to its proximity to Chinatown and many South Asians working in the nearby buildings, no study had focused on examining the mental health impact of the attack in this group. Based on data collected by the World Trade Center Health Registry from a sample of 4721 Asians 2-3 years after the disaster, this study provides a baseline investigation for the prevalence and the risk and protective factors for PTSD among Asian Americans directly exposed to the attack and compared this population against 42,862 non-Hispanic Whites. We found that Asians had a higher prevalence of PTSD compared to Whites (14.6 vs $11.7 \%)$. "Race-specific factors" significantly associated to PTSD in the multivariate analyses were noted among sociodemographics: higher education was protective for Whites but a risk factor for Asians; being employed was protective for Whites but had no effect for Asians; and being an immigrant was a risk factor for Whites but had no effect for Asians. However, income
\end{abstract}

W. W. Kung $(\varangle) \cdot$ P. Kim $\cdot$ X. Wang

Graduate School of Social Service, Fordham University, New York, NY, USA

e-mail: kung@fordham.edu

X. Liu $\cdot$ D. Huang $\cdot$ L. H. Yang

Mailman School of Public Health, Columbia University, New

York, NY, USA

L. H. Yang

College of Global Public Health, New York University, New York, NY, USA was a protective factor for both races. Other "universal factors" significantly increased the odds of PTSD symptoms but showed no racial differences, including exposure to the disaster and the presence of lower respiratory symptoms which intensified odds of PTSD by the greatest magnitude (3.6-3.9 times). Targeted effort to reach out to Asians is essential for prevention and follow up treatment given this group's striking history of underutilization of mental health services.

Keywords PTSD · 9/11 World Trade Center attack . Asian Americans · Mental health · Epidemiology · Social factors

\section{Introduction}

Due to the geographic location of Chinatown being near the World Trade Center (WTC) and the large number of South Asians working in the collapsed and nearby buildings [1], Asian Americans constituted a sizeable proportion of those exposed to the WTC attack on September 11, 2001 (9/11). Yet the number of studies that examined the Asian subgroup remains small. To our knowledge, of the less than ten studies that investigated Post Traumatic Stress Disorder (PTSD) among Asians, the number of participants included was small (from 8 to 148) $[2,3]$. The World Trade Center Health Registry (The Registry) is the only dataset that included a sizeable number of Asian Americans, with 4885 at baseline [4]. Among the Registry studies, race had been examined as one factor related to mental health outcomes, yet 
no studies have focused on the mental health outcomes of Asians and how risk and protective factors may differentially contribute to this group's outcomes. This is the first study to establish a baseline investigation to address this gap by focusing on mental health outcomes among Asians, using the non-Hispanic Whites (Whites) as a comparison group.

While existing epidemiological studies in community samples indicated that minorities generally demonstrated a higher risk for PTSD, Asians were found to have lower risk among minorities and compared to Whites [5, 6]. In WTC-related studies, the prevalence of PTSD among Asians presented a complex picture. Some found lower rates of PTSD among Asians compared to other races [7], while others found a higher rate [8].

Investigation of probable PTSD among Asians and Whites from the Registry would provide valuable clarification and elucidate how sociocultural factors impact the prevalence of PTSD, thus identifying vulnerable groups for prevention and treatment. This is especially important given the striking underutilization of mental health services among Asian Americans $[9,10]$ and to combat the popular perception of Asian Americans as a "model minority" who experience fewer mental health difficulties [11].

Because of the particular socioeconomic characteristics within the Asian communities, this racial group may be more vulnerable to having PTSD relative to Whites. Studies on Chinatown's economy indicated that years after the attack, small businesses that were central to the local economy (e.g., manufacturing and food services), and primary sources of employment, continued to struggle [12]. The "enclave effect" of the immigrant community further reinforced its isolation and hampered inflow of resources [13]. Prolonged economic hardship has been found to exacerbate any psychological distress [14], and low household income also contributed to higher PTSD prevalence [15].

Culture influences how mental health issues are experienced by shaping symptom manifestation when faced with trauma [16]. Due to the tendency in Asian culture to embody experiences of psychological distress [17], higher reports of somatic manifestation in PTSD symptoms such as heart pounding and difficulty breathing may occur. An increase in reports of the cooccurrence of lower respiratory symptoms (LRS) and PTSD have been noted in relation to the WTC attack $[18,19]$. By examining the association between somatic and psychological distresses, we may better comprehend the value of using physical health problems as an entry point to address mental health issues to circumvent Asians' tendency to avoid mental health services due to severe stigma around mental illness [18]. Comparison across race could also reflect on the extent to which culture may play in the association between psychological and physical distress.

We hypothesize that important 'race-specific' social statuses that predispose individuals to greater economic distress - such as loss of employment, lower income, and lower educational attainment - might lead to even more severe impacts on Asians. We expect higher reports of somatic symptoms related to PTSD, and greater association of LRS and PTSD among Asians given their higher tendency to express psychological distress in physical ailments. As context, we examine what we identify as 'universal' PTSD exposures to conditions known to contribute to disaster-related psychopathology such as proximity to disaster sites, witnessing of horror, and being physically injured [20]. Examining effects of these PTSD predictors among Asians using the Registry data provides the most comprehensive study on this issue to date, and provides valuable baseline data for future investigations.

\section{Method}

\section{WTC Registry Study}

The Registry, funded by the US Federal Emergency Management Agency, was developed in 2002 to evaluate the long-term health effects of the WTC attack [8]. Our study is based on baseline data which was collected from September 2003 to November 2004 (wave 1), 23 years after the disaster, from 71,433 total adult enrollees. Three additional waves of data were collected 5-6 years, 10-11 years, and 14-15 years afterwards. We focus on wave 1 data in this investigation since it captures the largest number of enrollees before they drop out (Asians had the highest rate of $46 \%$ among all racial groups, Whites was 24\%). To inform similar future disasters, wave 1 data, compared to those of later waves, also provide a more opportune time to target outreach efforts to Asians due to this group's reluctance to use mental health service [10], especially when the disaster becomes more distant. This baseline investigation also provides a valuable foundation for separate forthcoming 
investigations of longitudinal PTSD trajectories. Participants included rescue and recovery workers, workers in the WTC and nearby buildings, passersby, and residents in lower Manhattan. Full details are described elsewhere [21].

Institutional Review Board approvals were obtained from the first author's institution, Center for Disease Control and Prevention, and New York City Department of Health and Mental Hygiene.

\section{Study Participants}

In wave 1 , originally 4885 Asian and 43,712 White participants aged $\geq 18$ years were in the Registry. We excluded deceased enrollees whose information was provided by proxies (and, therefore, lacked information on PTSD symptoms), and individuals with pre-9/11 PTSD diagnosis, without imputable PCL score (see measures) and of an elderly age range (being $\geq 90$ years old). The final sample consisted of 4721 Asian and 42,862 White study participants.

\section{Measures}

PTSD To assess mental health impact, we examined PTSD using the PTSD Checklist, Civilian Version (PCL-C) [22] specifically worded about the 9/11 event. The scale is a self-report, 17 -item validated measure inquiring about symptomology in the last 30 days. Derived from the Diagnostic and Statistical Manual Edition IV (DSM-IV) [23], this measure has three clusters: intrusive and re-experiencing, numbing and avoidance, and hyperarousal symptoms. Responses ranged from "1" to "5"; higher scores indicated more PTSD symptomology. A cut-off score of $\geq 44^{1}$ was used, which demonstrated the highest levels of diagnostic efficiency (sensitivity $=94 \%$, specificity $=96 \%$, and diagnostic efficiency $=90 \%$ ) [24]. The scale has shown good psychometric properties among Asian Americans [25]. The items of physical reaction (e.g., heart pounding, trouble breathing and sweating when reminded of the disaster)

\footnotetext{
${ }^{1}$ For PCL scores with missing values, if the sum already exceeded 44, they were coded as having PTSD. Otherwise, imputation with the highest score (5) for the missing value was used. If the new score resulted in a sum lower than 44 , the participant was considered not to have PTSD; and if it was higher, it was considered missing. The final missing constituted $0.46 \%$ for Asians and $0.24 \%$ for Whites. This procedure was used to conservatively preserve cases who would not meet the cut-off for having PTSD and would otherwise be excluded from the study.
}

and sleep disturbance from moderate (3) to extreme (5) were used to denote somatic manifestations of PTSD.

Serious Psychological Distress (SPD) Our secondary mental health outcome, serious psychological distress, measured by the Kessler 6 scale, was used as a complement to the PCL. It is a screening tool for major mental illness [26] which has shown good psychometric properties among Asian Americans [27]. We adopted a cutoff score of 13 which indicated a sensitivity of 0.36 , specificity of 0.96 , and accuracy of 0.92 [28].

Exposure Exposure to the WTC disaster was examined using 6 dichotomous variables: 1 ) located in a collapsed/ damaged building during the attack; 2) dust cloud exposure; 3 ) witnessed $\geq 3$ horrific events (e.g., saw a plane hit a tower, someone falling from the tower, etc.); 4) sustained any injury (excluding eye irritation); 5) was a rescue, recovery or cleanup worker; 6) evacuated from residence for $\geq 48 \mathrm{~h}$.

Lower Respiratory Symptoms (LRS) This dichotomous variable indicating somatic distress was defined as positive if respondents reported new or worsening symptoms for one of the following: shortness of breath, persistent coughing, and wheezing since 9/11.

Recruitment Source Being self-identified or listidentified for recruitment was controlled for since the former tended to have more health concerns [29].

\section{Statistical Analyses}

We calculated summary statistics to describe participants' characteristics and the proportion of PTSD together with specific somatic symptom manifestations for the Asian and White groups, and compared across race using chi-square and $t$ tests. We examined the bivariate associations between each variable and PTSD by racial group using chi-square tests for the categorical variables and then examined for racial difference for the associations. We employed logistic regression models to investigate the associations between PTSD and the risk and protective factors of interest for each race. Adjusted odds ratio (AOR) and 95\% confidence interval (CI) were derived from the estimated model parameters. We used the Wald test to detect racial difference for each of the model parameters for associations between PCL and the predictors, and $p$ values were adjusted for 
multiple tests using the Hochberg method [30, 31] to control for family-wide error rate. We set the significance level at 0.05 . All analyses were conducted using SAS 9.4 [32].

\section{Results}

\section{Participants' Characteristics}

Table 1 shows the variable distribution of the characteristics of Asians and Whites. For sociodemographics, Asians had a larger proportion of middle-aged participants, about half were male, approximately $1 / 3$ were $<$ high-school educated, and another $1 / 3$ had a college degree. Their annual income distribution was skewed towards the lower spectrum of $<\$ 50,000$, over $1 / 4$ were unemployed, about $2 / 3$ were married/cohabiting, and over $1 / 3$ were immigrants. Compared to the White group, Asians had larger proportions of younger and older $(\geq 65)$ participants, had more females, and were somewhat less likely to be married/cohabiting. Asians were less educated overall, particularly having greater proportions of high-school graduates or less (30.1 vs $20.0 \%$ ), and lower proportions with "some college/ technical training" (13.6 vs. 22.9\%). Asians reported more than twice the proportion as Whites earning $<\$ 50,000$ (42.9 vs. $19.9 \%$ ). The unemployment rate among Asians nearly doubled that of Whites (27.6 vs. $14.0 \%$ ). Lastly, $1 / 3$ of Asians were immigrants, which contrasted with $5.4 \%$ of Whites. In sum, Asians generally had a poorer pattern of socioeconomic characteristics.

For disaster exposures, Asians and Whites had similar proportions of being "in collapsed/damaged buildings" (15.0 vs. $15.2 \%)$, "amidst dust cloud" (48.4 vs. $48.2 \%$ ), and "witnessing $\geq 3$ horrific events" (34.2 vs. $35.2 \%$ ). However, Asians had a larger proportion of being "evacuated from their residence" than Whites (4.3 vs. $2.6 \%$ ), while a smaller proportion was injured (12.9 vs. $16.8 \%$ ). Half of White participants were "rescue or recovery workers" compared to $15.4 \%$ for Asians.

For mental health conditions, Asians reported a higher proportion of having probable PTSD (14.6 vs. $11.7 \%$ in Whites, $p<0.0001)$ and Serious Psychological Distress (SPD; 8.0 vs. $5.7 \%$ in Whites). No racial difference was found for lower respiratory symptoms (LRS) between Asians and Whites (51.7 vs. 51.9\% Whites). For somatic manifestations within the PCL, a higher proportion of Asians had physical symptoms such as heart pounding and trouble breathing when reminded of the disaster compared to Whites (18.3 vs. $13.3 \%$, respectively, $p<0.0001)$. For sleep disturbance, Asians' higher proportion vs. Whites was marginal ( 28.8 vs. $27.5 \%$ respectively, $p=0.06$ ). The proportion of self-identified participants did not differ by race (70.2\% Asians vs. $69.2 \%$ Whites).

Bivariate Associations of Factors with Probable PTSD

The proportion of probable PTSD for each of the factors was reported in Table 2. For both races, higher proportions of probable PTSD were found in individuals with the following characteristics: middle aged, female, lower income, "divorced/separated/widowed", immigrants, "self-identified" participants, having experienced direct exposures of being "in collapsed/damaged buildings", "in dust cloud", "having witnessed $\geq 3$ horrific events" and "sustained injury"; and experiencing LRS and SPD. However, some racial differences in patterns of bivariate association were noted. Asians had higher proportions of probable PTSD than Whites in those whose ages were 45-64, those with higher education, higher income, employed, US-born, and those who were married/cohabiting. However, Asians who were "never married" had a lower proportion of probable PTSD than Whites. Striking racial contrasts were noted in education and current employment status, whereby generally speaking, less proportions of better-educated and employed Whites had PTSD compared with Asians. Higher proportions of immigrants had PTSD than their US-born counterparts for both races, but the difference was greater for Whites than Asians.

For relationships between disaster exposures and probable PTSD, Asians tended to have higher proportions of probable PTSD than Whites in all categories except for evacuated residence. SPD had a strong association with probable PTSD for both races although it was slightly stronger for Whites. Participants having LRS and being "self-identified" were highly associated with PTSD for both groups but showed no racial difference.

\section{Associations with PTSD in the Regression Model}

Table 3 presents covariate-adjusted odds ratios (AOR) for simultaneous effects of all factors of interest on probable PTSD for both races. Among Asians, logistic 
Table 1 Socio-demographics, disaster exposure and mental health outcome by race

\begin{tabular}{|c|c|c|c|c|}
\hline & $\begin{array}{l}\text { Factors at wave } 1 \\
\%(n)\end{array}$ & $\begin{array}{l}\text { Asian }(n=4721) \\
\%(n)\end{array}$ & White $(n=42,862)$ & $\begin{array}{l}\text { Racial difference } \\
p \text { value }\end{array}$ \\
\hline \multirow[t]{4}{*}{ Age } & $18-24$ & $6.25(295)$ & $3.42(1468)$ & \multirow[t]{4}{*}{$<0.0001$} \\
\hline & $25-44$ & $47.60(2247)$ & $49.16(21073)$ & \\
\hline & $45-64$ & $33.49(1581)$ & $42.71(18306)$ & \\
\hline & $65+$ & $12.67(598)$ & $4.70(2015)$ & \\
\hline \multirow[t]{2}{*}{ Gender } & Male & $51.79(2445)$ & $66.52(28512)$ & \multirow[t]{2}{*}{$<0.0001$} \\
\hline & Female & $48.21(2276)$ & $33.48(14350)$ & \\
\hline \multirow[t]{5}{*}{ Education } & High school or less & $30.10(1421)$ & $20.05(8595)$ & \multirow[t]{5}{*}{$<0.0001$} \\
\hline & Some college/technical school & $13.58(641)$ & $22.85(9795)$ & \\
\hline & College graduate & $34.38(1623)$ & $34.31(14704)$ & \\
\hline & Professional/graduate school & $20.97(990)$ & $22.44(9620)$ & \\
\hline & Missing & $0.97(46)$ & $0.35(148)$ & \\
\hline \multirow[t]{6}{*}{ Income } & $<\$ 50 \mathrm{k}$ & $42.89(2025)$ & $19.86(8514)$ & \multirow{6}{*}{$<0.0001$} \\
\hline & $\$ 50 \mathrm{k}-<\$ 5 \mathrm{k}$ & $14.09(665)$ & $18.92(8109)$ & \\
\hline & $\$ 75 \mathrm{k}-<\$ 100 \mathrm{k}$ & $11.14(526)$ & $18.96(8127)$ & \\
\hline & $\$ 100 \mathrm{k}-<\$ 150 \mathrm{k}$ & $9.60(453)$ & $17.37(7447)$ & \\
\hline & $\$ 150 \mathrm{k}$ or more & $8.05(380)$ & $14.01(6005)$ & \\
\hline & Missing & $14.23(672)$ & $10.87(4660)$ & \\
\hline \multirow[t]{2}{*}{ Employment status } & Unemployed or unreported & $27.62(1304)$ & $14.01(6005)$ & \multirow[t]{2}{*}{$<0.0001$} \\
\hline & Employed & $72.38(3417)$ & 85.99 (36857) & \\
\hline \multirow[t]{4}{*}{ Marital status } & Married or Living with Partner & $65.30(3083)$ & $68.09(29185)$ & \multirow[t]{4}{*}{$<0.0001 *^{\mathrm{a}}$} \\
\hline & Divorced/widowed/separated & $11.14(526)$ & $10.93(4685)$ & \\
\hline & Never married & $22.45(1060)$ & $20.58(8820)$ & \\
\hline & Missing & $1.10(52)$ & $0.40(172)$ & \\
\hline \multirow[t]{2}{*}{ Immigration status } & US born & $63.46(2996)$ & $94.59(40543)$ & \multirow[t]{2}{*}{$<0.0001$} \\
\hline & Immigrant or unreported & $36.54(1725)$ & $5.41(2319)$ & \\
\hline \multirow[t]{2}{*}{ In collapsed/damaged building } & No & $84.98(4012)$ & $84.80(36349)$ & \multirow[t]{2}{*}{0.7473} \\
\hline & Yes & $15.02(709)$ & $15.20(6513)$ & \\
\hline \multirow[t]{2}{*}{ In dust cloud } & No & $51.56(2434)$ & $51.81(22206)$ & \multirow[t]{2}{*}{0.7430} \\
\hline & Yes & $48.44(2287)$ & $48.19(20656)$ & \\
\hline \multirow[t]{2}{*}{ Witnessed $\geq 3$ horrific $9 / 11$ events } & No & $65.77(3105)$ & $64.83(27789)$ & \multirow[t]{2}{*}{0.2007} \\
\hline & Yes & $34.23(1616)$ & $35.17(15073)$ & \\
\hline \multirow[t]{2}{*}{ Sustained an injury (not eye) } & No & $87.08(4111)$ & $83.24(35677)$ & \multirow[t]{2}{*}{$<0.0001$} \\
\hline & Yes & $12.92(610)$ & $16.76(7185)$ & \\
\hline \multirow[t]{2}{*}{ Rescue/recovery worker } & No & $84.56(3992)$ & $49.85(21365)$ & $<0.0001$ \\
\hline & Yes & $15.44(729)$ & $50.15(21497)$ & \\
\hline Evacuated Residence for $\geq 48 \mathrm{~h}$ & No & $95.66(4516)$ & 97.39 (41744) & $<0.0001$ \\
\hline & Yes & $4.34(205)$ & $2.61(1118)$ & \\
\hline Probable PTSD $(\mathrm{PCL} \geq 44)$ & No & $85.36(4030)$ & $88.30(37849)$ & $<0.0001$ \\
\hline & Yes & $14.64(691)$ & $11.70(5013)$ & \\
\hline SPD & No & $90.43(4269)$ & $94.02(40297)$ & $<0.0001$ \\
\hline & Yes & $8.01(378)$ & $5.66(2427)$ & \\
\hline & Missing & $1.57(74)$ & $0.32(138)$ & \\
\hline LRS & No & $46.05(2174)$ & $46.80(20059)$ & $<0.0001 *^{b}$ \\
\hline & Yes & $51.71(2441)$ & $51.85(22223)$ & \\
\hline & Missing & 2.25 (106) & $1.35(580)$ & \\
\hline PCL: physical symptoms & No & $81.38(3842)$ & $86.62(37129)$ & $<0.0001$ \\
\hline & Yes & $18.17(858)$ & $13.28(5694)$ & \\
\hline
\end{tabular}


Table 1 (continued)

\begin{tabular}{|c|c|c|c|c|}
\hline & $\begin{array}{l}\text { Factors at wave } 1 \\
\%(n)\end{array}$ & $\begin{array}{l}\text { Asian }(n=4721) \\
\%(n)\end{array}$ & White $(n=42,862)$ & $\begin{array}{l}\text { Racial difference } \\
p \text { value }\end{array}$ \\
\hline \multirow{3}{*}{ PCL: trouble sleeping } & Missing & $0.44(21)$ & $0.09(39)$ & \multirow[b]{2}{*}{0.0602} \\
\hline & $\begin{array}{l}\text { No } \\
\text { Yes }\end{array}$ & $\begin{array}{l}71.07(3355) \\
28.77(1358)\end{array}$ & $\begin{array}{l}72.41(31036) \\
27.49(11784)\end{array}$ & \\
\hline & Missing & $0.17(8)$ & $0.10(42)$ & \multirow[b]{2}{*}{0.1544} \\
\hline Recruitment source & $\begin{array}{l}\text { List-identified } \\
\text { Self-identified }\end{array}$ & $\begin{array}{l}29.80(1407) \\
70.20(3314)\end{array}$ & $\begin{array}{l}30.81(13206) \\
69.19(29656)\end{array}$ & \\
\hline
\end{tabular}

${ }^{*} p$ value changed to $>0.0001$ after excluding missing values: ${ }^{\mathrm{a}} p=0.0024,{ }^{\mathrm{b}} p=0.6664$

regression analyses revealed that probable PTSD was related to the socio-demographic variables of age, sex, education, income, and marital status. Being in the youngest and the oldest group compared to the prime years of 25-44 were significantly protective, while being female, having low income, having "some college/ technical training", and being a "college graduate" were at higher risk. Compared to those married/cohabiting, the odds of probable PTSD was higher for "divorced/ separated/widowed" individuals while lower for those "never married". Immigration status and recruitment source were unrelated to probable PTSD. LRS and the exposure variables of injury, having "witnessed $\geq 3$ horrific events," and "in dust cloud" increased the odds of probable PTSD in Asians.

Among Whites, probable PTSD was related to all sociodemographic variables, all WTC exposures except having "evacuated for $\geq 48 \mathrm{~h}$ ", recruitment source, and LRS. Comparing between the races, after adjusting the $p$ values to control for family wide error rate, we found education, current employment, and immigration status to be significantly different at the 0.05 level in their associations with probable PTSD. For Whites, higher education was clearly protective, with a risk reduction for "college graduates" $(\mathrm{AOR}=0.58, \mathrm{CI}=0.53-0.64)$ and "graduate training" $(\mathrm{AOR}=0.49, \mathrm{CI}=0.44-0.54)$ relative to high-school educated or less. However, for Asians, several higher education categories became risk factors including "some college/technical training" $(\mathrm{AOR}=1.33, \mathrm{CI}=1.03-1.71)$ and "college graduates" (AOR $=1.40, \mathrm{CI}=1.03-1.89)$. The effect of being employed continued to be contrasted between racesbeing employed had no significant impact on Asians (AOR $=0.83, \mathrm{CI}=0.66-1.04)$ but attenuated PTSD risk by almost half for Whites $(\mathrm{AOR}=0.53, \mathrm{CI}=0.49$ -
0.58). Finally, being an immigrant remained a risk factor for Whites $(\mathrm{AOR}=1.61, \mathrm{CI}=1.42-1.82)$ but not for Asians (AOR $=0.94, \mathrm{CI}=0.78-1.13)$.

While being "divorced/separated/widowed" increased the risk of PTSD compared to those married/ cohabiting for both Asians and Whites (by 44 and 50\% respectively), "never married" was protective for Asians only (AOR $=0.65, \mathrm{CI}=0.50-0.86$ ).

The highest impact from exposure for both races came from injury (AOR $=2.69, \mathrm{CI}=2.16-3.33$ for Asians; $\mathrm{AOR}=2.21, \mathrm{CI}=2.05-2.37$ for Whites). Having "witnessed $\geq 3$ horrific events" and "being caught in the dust cloud" were also significant for both races. "Being rescue or recovery workers" attenuated the risk for Whites significantly though did not reach significance for Asians. None of the exposure factors showed racial differences. Of all the factors, having LRS showed the greatest odds for PTSD $(\mathrm{AOR}=3.55, \mathrm{CI}=2.86-$ 4.41 for Asians; $\mathrm{AOR}=3.86, \mathrm{CI}=3.56-4.19$ for Whites), but with no significant racial difference. Selfidentified White participants showed slightly higher risk for PTSD but not for Asians.

\section{Discussion}

Study Contribution

The current study is unique from other Registry studies in that it focuses on addressing the mental health impact of the WTC attack among Asian Americans specifically, with an emphasis on understanding the significant factors affecting PTSD in Asians. Also, unlike other studies on Asians [33, 34], we used Whites as an explicit reference group. 
Table 2 Proportion of probable PTSD (PCL $\geq 44$ ) by levels of factors in Asian Americans and in Whites.

\begin{tabular}{|c|c|c|c|c|c|c|}
\hline \multirow[t]{2}{*}{ Factors at wave 1} & & \multicolumn{2}{|l|}{ Asian $(n=4721)$} & \multicolumn{2}{|l|}{ White $(n=42,862)$} & \multirow{2}{*}{$\begin{array}{l}\text { Racial } \\
\text { difference } \\
p \text { value }\end{array}$} \\
\hline & & $\begin{array}{l}\% \text { Probable PTSD } \\
(n)\end{array}$ & $p$ value & $\begin{array}{l}\% \text { Probable PTSD } \\
\text { (n) }\end{array}$ & $p$ value & \\
\hline \multirow[t]{3}{*}{ Age } & $\begin{array}{l}18-24 \\
25-44\end{array}$ & $\begin{array}{l}4.07(12) \\
13.22(297)\end{array}$ & \multirow[t]{3}{*}{$<0.0001$} & $\begin{array}{l}8.45(124) \\
11.24(2368)\end{array}$ & \multirow[t]{3}{*}{$<0.0001$} & \multirow[t]{3}{*}{$<0.0001$} \\
\hline & $45-64$ & $21.25(336)$ & & $12.73(2330)$ & & \\
\hline & $65-89$ & $7.69(46)$ & & $9.48(191)$ & & \\
\hline Gender & $\begin{array}{l}\text { Male } \\
\text { Female }\end{array}$ & $\begin{array}{l}13.29(325) \\
16.08(366)\end{array}$ & 0.0068 & $\begin{array}{l}10.28(2932) \\
14.50(2081)\end{array}$ & $<0.0001$ & 0.0552 \\
\hline Education & $\begin{array}{l}\text { High school or less } \\
\text { Some college/technical } \\
\text { school } \\
\text { College graduate } \\
\text { Professional/graduate school } \\
\text { Missing }\end{array}$ & $\begin{array}{l}14.36(204) \\
17.32(111) \\
15.22(247) \\
12.42(123) \\
13.04(6)\end{array}$ & $0.0850 *^{\mathrm{a}}$ & $\begin{array}{l}17.36(1492) \\
12.84(1258) \\
10.03(1475) \\
7.96(766) \\
14.86(22)\end{array}$ & $<0.0001$ & $<0.0001$ \\
\hline Income & $\begin{array}{l}<\$ 50 \mathrm{k} \\
\$ 50 \mathrm{k}-<\$ 75 \mathrm{k} \\
\$ 75 \mathrm{k}-<\$ 100 \mathrm{k} \\
\$ 100 \mathrm{k}-<\$ 150 \mathrm{k} \\
\$ 150 \mathrm{k} \text { or more } \\
\text { Missing }\end{array}$ & $\begin{array}{l}17.43(353) \\
15.34(102) \\
13.12(69) \\
13.25(60) \\
8.42(32) \\
11.16(75)\end{array}$ & $<0.0001$ & $\begin{array}{l}18.10(1541) \\
13.12(1064) \\
10.35(841) \\
8.78(654) \\
7.59(456) \\
9.81(457)\end{array}$ & $<0.0001$ & 0.0192 \\
\hline Employment Status & $\begin{array}{l}\text { Unemployed or unreported } \\
\text { Employed }\end{array}$ & $\begin{array}{l}13.88(181) \\
14.93(510)\end{array}$ & 0.3637 & $\begin{array}{l}19.18(1152) \\
10.48(3861)\end{array}$ & $<0.0001$ & $<0.0001$ \\
\hline Marital status & $\begin{array}{l}\text { Married or living with } \\
\text { partner } \\
\text { Divorced/widowed/separated } \\
\text { Never married } \\
\text { Missing }\end{array}$ & $\begin{array}{l}15.34(473) \\
21.10(111) \\
9.34(99) \\
15.38(8)\end{array}$ & $<0.0001$ & $\begin{array}{l}10.14(2960) \\
20.36(954) \\
12.15(1072) \\
15.70(27)\end{array}$ & $<0.0001$ & $<0.0001$ \\
\hline Immigration status & $\begin{array}{l}\text { US born } \\
\text { Immigrant or unreported }\end{array}$ & $\begin{array}{l}13.58(407) \\
16.46(284)\end{array}$ & 0.0070 & $\begin{array}{l}11.35(4603) \\
17.68(410)\end{array}$ & $<0.0001$ & 0.0041 \\
\hline $\begin{array}{l}\text { In collapsed/damaged } \\
\text { building }\end{array}$ & $\begin{array}{l}\text { No } \\
\text { Yes }\end{array}$ & $\begin{array}{l}13.73(551) \\
19.75(140)\end{array}$ & $<0.0001$ & $\begin{array}{l}10.94(3978) \\
15.89(1035)\end{array}$ & $<0.0001$ & 0.9628 \\
\hline In dust cloud & $\begin{array}{l}\text { No } \\
\text { Yes }\end{array}$ & $\begin{array}{l}9.16(223) \\
20.46(468)\end{array}$ & $<0.0001$ & $\begin{array}{l}7.47(1658) \\
16.24(3355)\end{array}$ & $<0.0001$ & 0.5209 \\
\hline $\begin{array}{l}\text { Witnessed } \geq 3 \text { horrific } \\
\text { events }\end{array}$ & $\begin{array}{l}\text { No } \\
\text { Yes }\end{array}$ & $\begin{array}{l}10.05(312) \\
23.45(379)\end{array}$ & $<0.0001$ & $\begin{array}{l}7.94(2206) \\
18.62(2807)\end{array}$ & $<0.0001$ & 0.7111 \\
\hline $\begin{array}{l}\text { Sustained an injury (not } \\
\text { eye) }\end{array}$ & $\begin{array}{l}\text { No } \\
\text { Yes }\end{array}$ & $\begin{array}{l}11.41(469) \\
36.39(222)\end{array}$ & $<0.0001$ & $\begin{array}{l}8.84(3153) \\
25.89(1860)\end{array}$ & $<0.0001$ & 0.0416 \\
\hline Rescue/recovery worker & $\begin{array}{l}\text { No } \\
\text { Yes }\end{array}$ & $\begin{array}{l}14.63(584) \\
14.68(107)\end{array}$ & 0.9729 & $\begin{array}{l}12.94(2764) \\
10.46(2249)\end{array}$ & $<0.0001$ & 0.0381 \\
\hline $\begin{array}{l}\text { Evacuated residence for } \\
\quad \geq 48 \mathrm{~h}\end{array}$ & $\begin{array}{l}\text { No } \\
\text { Yes }\end{array}$ & $\begin{array}{l}14.81(669) \\
10.73(22)\end{array}$ & 0.1058 & $\begin{array}{l}11.65(4865) \\
13.24(148)\end{array}$ & 0.1040 & 0.0366 \\
\hline SPD & $\begin{array}{l}\text { No } \\
\text { Yes } \\
\text { Missing }\end{array}$ & $\begin{array}{l}8.92(381) \\
73.81(279) \\
41.89(31)\end{array}$ & $<0.0001$ & $\begin{array}{l}7.59(3059) \\
78.45(1904) \\
36.23(50)\end{array}$ & $<0.0001$ & 0.0072 \\
\hline LRS & $\begin{array}{l}\text { No } \\
\text { Yes } \\
\text { Missing }\end{array}$ & $\begin{array}{l}5.80(126) \\
22.49(549) \\
15.09(16)\end{array}$ & $<0.0001$ & $\begin{array}{l}4.24(851) \\
18.50(4111) \\
8.79(51)\end{array}$ & $<0.0001$ & 0.4054 \\
\hline Recruitment source & $\begin{array}{l}\text { List-identified } \\
\text { Self-identified }\end{array}$ & $\begin{array}{l}12.15(171) \\
15.69(520)\end{array}$ & 0.0017 & $\begin{array}{l}8.58(1133) \\
13.08(3880)\end{array}$ & $<0.0001$ & 0.0815 \\
\hline
\end{tabular}

$* p$ value changed to $<0.05$ after excluding missing values: ${ }^{a} p=0.0443$ 
Table 3 Adjusted odds ratio (AOR) and 95\% confidence interval for association between predictors and probable PTSD (PCL $\geq 44$ ), by race.

\begin{tabular}{|c|c|c|c|c|}
\hline Factors at wave 1 & & $\begin{array}{l}\text { Asian } \\
(n=4721) \\
\text { AOR }(95 \% \text { CI })\end{array}$ & $\begin{array}{l}\text { White } \\
(n=42,862) \\
\text { AOR }(95 \% \text { CI) }\end{array}$ & $\begin{array}{l}\text { Racial } \\
\text { difference } \\
* p \text { value }\end{array}$ \\
\hline \multirow[t]{4}{*}{ Age } & $18-24$ & $0.34(0.18-0.66)$ & $0.55(0.44-0.68)$ & 0.0624 \\
\hline & $25-44$ & 1 & 1 & \\
\hline & $45-64$ & $1.41(1.16-1.73)$ & $1.04(0.97-1.12)$ & \\
\hline & $65+$ & $0.39(0.26-0.58)$ & $0.43(0.36-0.52)$ & \\
\hline \multirow[t]{2}{*}{ Gender } & Male & 1 & 1 & 0.0902 \\
\hline & Female & $1.21(1.01-1.46)$ & $1.44(1.34-1.55)$ & \\
\hline \multirow[t]{5}{*}{ Education } & High school or less & 1 & 1 & 0.0013 \\
\hline & Some college/technical school & $1.40(1.03-1.89)$ & $0.69(0.63-0.76)$ & \\
\hline & College graduate & $1.33(1.03-1.72)$ & $0.58(0.53-0.64)$ & \\
\hline & Professional/graduate school & $1.09(0.81-1.49)$ & $0.49(0.44-0.54)$ & \\
\hline & Missing & $1.19(0.44-3.24)$ & $0.70(0.41-1.19)$ & \\
\hline \multirow[t]{6}{*}{ Income } & $<\$ 50 \mathrm{k}$ & 1 & 1 & 0.9964 \\
\hline & $\$ 50 \mathrm{k}-<\$ 75 \mathrm{k}$ & $0.68(0.51-0.90)$ & $0.70(0.64-0.77)$ & \\
\hline & $\$ 75 \mathrm{k}-<\$ 100 \mathrm{k}$ & $0.51(0.37-0.70)$ & $0.56(0.50-0.62)$ & \\
\hline & $\$ 100 \mathrm{k}-<\$ 150 \mathrm{k}$ & $0.54(0.38-0.77)$ & $0.50(0.44-0.56)$ & \\
\hline & $\$ 150 \mathrm{k}$ or more & $0.37(0.24-0.56)$ & $0.48(0.43-0.55)$ & \\
\hline & Missing & $0.65(0.49-0.88)$ & $0.55(0.49-0.63)$ & \\
\hline \multirow[t]{2}{*}{ Employment status } & Unemployed/unreported & 1 & 1 & 0.0052 \\
\hline & Employed & $0.83(0.66-1.04)$ & $0.53(0.49-0.58)$ & \\
\hline \multirow[t]{4}{*}{ Marital status } & Married or Living with Partner & 1 & 1 & 0.1390 \\
\hline & Divorced/widowed & $1.44(1.11-1.89)$ & $1.50(1.37-1.65)$ & \\
\hline & Never married & $0.65(0.50-0.86)$ & $1.06(0.97-1.15)$ & \\
\hline & Missing & $1.29(0.54-3.04)$ & $1.48(0.91-2.40)$ & \\
\hline \multirow[t]{2}{*}{ Immigration status } & US born & 1 & 1 & 0.0013 \\
\hline & Immigrant or unreported & $0.94(0.78-1.13)$ & $1.61(1.42-1.82)$ & \\
\hline \multirow[t]{2}{*}{ In collapsed/damaged building } & No & 1 & 1 & 0.9964 \\
\hline & Yes & $1.26(0.98-1.61)$ & $1.25(1.14-1.36)$ & \\
\hline \multirow[t]{2}{*}{ In dust cloud } & No & 1 & 1 & 0.4347 \\
\hline & Yes & $1.52(1.24-1.85)$ & $1.23(1.14-1.32)$ & \\
\hline \multirow[t]{2}{*}{ Witnessed $\geq 3$ horrific events } & No & 1 & 1 & 0.9964 \\
\hline & Yes & $1.85(1.53-2.24)$ & $1.84(1.71-1.98)$ & \\
\hline \multirow[t]{2}{*}{ Sustained an injury (not eye) } & No & 1 & 1 & 0.7296 \\
\hline & Yes & $2.69(2.16-3.33)$ & $2.21(2.05-2.37)$ & \\
\hline \multirow[t]{2}{*}{ Rescue/recovery worker } & No & 1 & 1 & 0.9964 \\
\hline & Yes & $0.94(0.72-1.22)$ & $0.83(0.76-0.89)$ & \\
\hline \multirow[t]{2}{*}{ Evacuated residence for $\geq 48 \mathrm{~h}$} & No & 1 & 1 & 0.9964 \\
\hline & Yes & $0.82(0.50-1.34)$ & $1.16(0.95-1.41)$ & \\
\hline \multirow[t]{3}{*}{ LRS } & No & 1 & 1 & 0.9964 \\
\hline & Yes & $3.55(2.86-4.41)$ & $3.86(3.56-4.19)$ & \\
\hline & Missing & $2.90(1.61-5.20)$ & $1.76(1.29-2.39)$ & \\
\hline \multirow[t]{2}{*}{ Recruitment source } & List-identified & 1 & 1 & 0.9964 \\
\hline & Self-identified & $1.09(0.88-1.34)$ & $1.09(1.01-1.18)$ & \\
\hline
\end{tabular}

*The $p$ values for racial difference were adjusted for multiple tests using Hochberg method to control for family-wide error rate

This investigation examined participants across different eligibility groups who were exposed, such as responders or residents of Lower Manhattan, thereby providing a more comprehensive picture of affected populations within the two races.
Mental Health Impact of the WTC Attack by Race

Asians reported a significantly higher proportion of having probable PTSD (14.6\%) compared to Whites $(11.7 \%)$, with a similar pattern noted in SPD (8.0 vs 
5.7\%). The PTSD rate of Asians was comparable to the $15 \%$ reported among the civilian survivors from the two collapsed WTC buildings [35], and higher than the rescue and recovery workers (12.4\%) [36] indicating a very high mental health impact on this group. Our study supports the general finding in other Registry studies that being Asian was a risk factor for PTSD compared to Whites, e.g. among male police responders [37], civilian survivors of the collapsed WTC buildings [35], and residents, office workers, and passersby [18]. However, our findings differ from other WTC studies in which the broader New York City population was randomly sampled whereby being Asian was protective from developing PTSD [7, 38]. The broader sampling frames of these studies, which included individuals not directly exposed to the attack and the Asian participants sampled being more acculturated as indicated in the language interviewed [38] may account for the difference. Our findings also revealed a very different picture from large-scale epidemiological studies (e.g., the National Epidemiologic Survey on Alcohol and Related Conditions [6]; and the Collaborative Psychiatric Epidemiology Studies [5]) in which lifetime PTSD and the risk of developing PTSD after a traumatic event were the lowest in Asians among all races. Our study, which has the methodological advantage of comparing a sizeable sample of Asian Americans and Whites following the same traumatic event, thus challenges the "model minority" myth of Asians having better mental health relative to other races [11].

\section{'Race-Specific' Differential Association of Socioeconomic Status with PTSD}

Asian participants clearly came from significantly more disadvantaged socioeconomic background than Whites - having lower education and income, almost twice as many unemployed (27.6 vs $14.0 \%$ ), more than double being 65 years or older (12.7 vs $4.7 \%$ ), and having nearly seven times more immigrants (36.5 vs $5.4 \%$ ) - may make them more susceptible to the tremendous stresses with less access to resources following the disaster. The higher percentage of males among Whites (66.5 vs $51.8 \%$ ) could be explained by their higher proportion of rescue and recovery workers compared with Asians (50.2 vs $15.4 \%$ ), which might also, in part, explain their higher employment rate in our sample given their secure job situations compared to affected small businesses in Chinatown.
Some sociodemographic variables that were directly related to economic resources showed striking racial contrasts in their association with PTSD. Our current findings among Whites coincided with previous studies on Whites that low socioeconomic status was associated with high PTSD [39, 40]. Higher education for Whites clearly was protective (risk reduction of $31-42 \%$ for "college or higher education" relative to high school). In contrast, Asians who had "some college/technical training" or more fared worse (a risk increase of 33$40 \%$ ), while those with professional or graduate school training exhibited no difference when compared to high school education. Among Whites, more education might be associated with more resources in terms of knowledge and social capital to protect against the aftereffects of the trauma, which may be harder to attain for Asians, many of whom are immigrants who are less integrated into the mainstream society. Further, it is well known that immigrants' educational attainments often do not positively correlate with economic or occupational accomplishments, partly because their qualifications and work experiences are not recognized in their host countries [41, 42].

For Asians, being employed was not related to PTSD, but for Whites it attenuated the risk for PTSD by almost half. Being employed may not confer the same protection on Asians as it does for Whites, likely due to underemployment and the menial nature of the jobs of this group (e.g., restaurant or other service workers), as indicated by a much greater proportion of Asians earning $<\$ 50,000$ household income compared to Whites (42.9 vs 19.9\%). Regardless of employment status, financial impacts on Asians was reported to be large as reflected in a study of the displaced Chinese garment workers where $81 \%$ reporting lowered income 8 months after 9/11 [33]. This interpretation regarding the importance of having economic resources is further bolstered by income playing a clearly protective role against PTSD in many other WTC studies $[8,15]$.

Finally, although we were unable to directly test this, we speculate that the larger Asian immigrant communities in New York City likely provided more social support than was available for White immigrants so that the immigration status posed higher risk for Whites while it had no significant impact on Asians when other factors such as income were controlled for. Furthermore, it is possible that the more collective Asian culture provided a larger social network of relatives, clansmen, and friends lending more social support [43]. 
'Universal' Impact of Direct Exposure and LRS on PTSD

As expected, direct exposure to the attack when individuals were in imminent danger - such as "being in dust cloud", "in collapsed/damaged building", having "witnessed horrific events", and having "sustained an injury" - raised the risk of PTSD (an increase of $23 \%$ to $270 \%$ based on the AORs) with no racial differences. However, although being rescue or recovery workers could have exposed individuals to higher risk, their training might have better prepared them to deal with such impact [37], resulting in this being more of a protective factor which attained significance for Whites but not Asians (although the AOR in Asians was protective in nature). "Having been evacuated" for $\geq 48 \mathrm{~h}$ was not significantly associated with PTSD for both races. These results signal a relatively consistent role of direct exposure enhancing the risk of PTSD in a similar fashion across both racial groups.

The association of LRS with PTSD was found to be strong in both races as reported in other Registry studies $[19,44]$. However, contrary to our hypothesis that Asians would show a greater tendency than Whites in the association between somatic and psychological symptoms, no racial difference was noted, despite the higher proportion of disaster-related somatic manifestation of PTSD symptoms among Asians than Whites. Perhaps the mutually reinforcing nature of the PTSD and LRS resulted in an intensification of symptoms of both illnesses [19] thus outweighing Asian culture's tendency to focus on their somatic expression of distress and to downplay their psychological distress to avoid stigma.

\section{Limitations \& Strengths}

Study weaknesses include not capturing the diversity in ethnicity within the Asian group, although these groups do share some similar characteristics [42]. Our study's cross-sectional nature precluded us from drawing causal relationships, e.g., income or employment status could result from PTSD rather than being its cause, but our next longitudinal studies would attempt to address this issue. Some relevant variables such as social support, post 9/11 trauma or stress, and mental health service use that could help explain PTSD prevalence are available only in later waves of the study. The main mental health outcome measure (PCL) was based on respondents' self-report instead of clinician diagnosis which is considered the gold standard, but the adherence of which is difficult given the large scale of the Registry study. Further, 2-3 years after the disaster, respondents might exhibit a recall bias, and an avoidance tendency could lower the accuracy of self-report of the exposure [8] leaning towards under-reporting.

However, we believe these limitations are outweighed by the study strengths. This is the largest single study examining the mental health impact on Asian Americans directly affected 2-3 years after the WTC attack. The separate analyses for racial groups facilitated the identification of PTSD risk and protective factors that were specific to the Asian community. This study also shifted the focus to more recent postmigration traumatic experiences, thus providing a valuable update to previous studies on Asian Americans that focused on pre-migration war experiences and postmigration violence, e.g. among Cambodians [45].

\section{Study Implications}

The overall vulnerability for Asians, in terms of their more disadvantaged positions and their high proportion of probable PTSD, signals needed attention and help. Targeted efforts to reach out to this economically disadvantaged population to ensure follow up treatment is very important both due to their high risk for PTSD and other psychological distress, and their low tendency to seek and sustain treatment effort [10]. Our study findings suggest ways to identify and target higher-risk Asian groups. The large percentage of individuals from very low household income needs to be targeted for outreach. The level of employment and earned income is important in identifying Asians at risk for PTSD, not just employment status alone. Although the association between LRS and PTSD for Asians was not higher than Whites, the heightened risk by $360 \%$ for Asians with LRS, and higher tendency to manifest somatic symptoms of psychological distress such as heart pounding, trouble breathing and sweating offers an important inroad to screen for potential PTSD given the population's tendency to seek help for physical ailments [17]. An effective "bridge" between medical and psychiatric services whereby both services are available on one site could help to reduce the stigma and ensure service utilization [46]. Likewise, targeting those with direct WTC exposure is essential given the higher risk for 
PTSD. Outreach efforts such as The Registry's Treatment Referral Program should be continued and staffed with bilingual personnel given the high percentage of Asians being immigrants (76.4\%) [47] and many were not proficient in English [48]. Bilingual services are also needed to engage this population in treatment. We hope that these findings will serve as a call to action to address the mental health risks of this group that likely remain in great need of help due to the lingering nature of PTSD [36].

Acknowledgements This study was supported by CDC National Institute of Occupational Safety and Health (NIOSH), for the grant titled Mental Health Impact and Service Use among Asian Survivors and Rescuers Exposed to the WTC Attack, \#1U01 OH01051601A1. We would also like to thank the World Trade Center Health Registry for offering access to their dataset.

\section{References}

1. Asian American Federation of New York. Asian American mental health: a post September 11th needs assessment. 2003. http://www.aafny.org/doc/AsianAmericanMentalHealth.pdf. Accessed 1 October, 2013.

2. Constantine MG, Alleyne VL, Caldwell LD, McRae MB, Suzuki LA. Coping responses of Asian, Black, and Latino/ Latina New York City residents following the September 11, 2001 terrorist attacks against the United States. Cult Divers Ethn Minor Psychol. 2005;11(4):293-308. https://doi. org/10.1037/1099-9809.11.4.293.

3. Inman AG, Yeh CJ, Madan-Bahel A, Nath S. Bereavement and coping of South Asian families post 9/11. J Multicult Couns Dev. 2007;35(2):101-15. https://doi.org/10.1002 j.2161-1912.2007.tb00053.x.

4. New York City Health Department. 9/11 Health. 2016; http://www.nyc.gov/html/doh/wtc/html/home/home.shtml. Accessed 13 March 2016.

5. Asnaani A, Richey JA, Dimaite R, Hinton DE, Hofmann SG. A cross-ethnic comparison of lifetime prevalence rates of anxiety disorders. J Nerv Ment Dis. 2010;198(8):551-5. https://doi.org/10.1097/NMD.0b013e3181ea169f.

6. Roberts AL, Gilman SE, Breslau J, Breslau N, Koenen KC. Race/ethnic differences in exposure to traumatic events, development of post-traumatic stress disorder, and treatment-seeking for post-traumatic stress disorder in the United States. Psychol Med. 2011;41(1):71-83. https://doi. org/10.1017/S0033291710000401.

7. Bonanno GA, Galea S, Bucciarelli A, Vlahov D. What predicts psychological resilience after disaster? The role of demographics, resources, and life stress. J Consult Clin Psychol. 2007;75(5):671-82. https://doi.org/10.1037/0022006X.75.5.671.

8. Farfel M, DiGrande L, Brackbill R, Prann A, Cone J, Friedman S, et al. An overview of 9/11 experiences and respiratory and mental health conditions among World
Trade Center Health Registry enrollees. J Urban Health. 2008;85(6):880-909. https://doi.org/10.1007/s11524-0089317-4.

9. Abe-Kim J, Takeuchi DT, Seunghye H, et al. Use of mental health-related services among immigrant and US-born Asian Americans: results from the National Latino and Asian American study. Am J Public Health. 2007;97(1): 91-8. https://doi.org/10.2105/AJPH.2006.098541.

10. U.S. Department of Health and Human Services. Mental health: culture, race, and ethnicity: a supplement to mental health, a report of the surgeon general : executive summary. Washington, D.C.2001.

11. Sue S, Chu JP, Cheng JKY, Saad CS. Asian American mental health: a call to action. Am Psychol. 2012;67(7): 532-44. https://doi.org/10.1037/a0028900.

12. Asian American Federation. Revitalizing Chinatown businesses: challenges and opportunities. 2008. http://www. aafny.org/publications.asp. Accessed October 1, 2013.

13. Chin M. Chinese garment workers after $9 / 11$. In: Foner N, editor. Wounded City: the social impact of 9/11 on New York City. New York: Russell Sage Foundation; 2005. p. 184207.

14. Boscarino JA, Adams RE. PTSD onset and course following the World Trade Center disaster: findings and implications for future research. Soc Psychiatry Psychiatr Epidemiol. 2009;44(10):887-98. https://doi.org/10.1007/s00127-0090011-y.

15. DiGrande L, Thorpe LE, Wu D, et al. Posttraumatic stress symptoms, PTSD, and risk factors among lower Manhattan residents 2-3 years after the September 11, 2001 terrorist attacks. J Trauma Stress. 2008;21(3):264-73. https://doi. org/10.1002/jts.20345.

16. Norris FH, Alegria M. Mental health care for ethnic minority individuals and communities in the aftermath of disasters and mass violence. CNS Spectrums. 2005;10(2):132-40. https://doi.org/10.1017/S1092852900019477.

17. Kung WW, Lu PC. How symptom manifestations affect help seeking for mental health problems among Chinese Americans. J Nerv Ment Dis. 2008;196(1):46-54. https://doi.org/10.1097/NMD.0b013e31815fa4f9.

18. Nair HP, Ekenga CC, Cone JE, Brackbill RM, Farfel MR, Stellman SD. Co-occurring lower respiratory symptoms and posttraumatic stress disorder 5 to 6 years after the World Trade Center terrorist attack. Am J Public Health. 2012;102(10):1964-73. https://doi.org/10.2105 /AJPH.2012.300690.

19. Friedman SM, Farfel MR, Maslow CB, Cone JE, Brackbill RM, Stellman SD. Comorbid persistent lower respiratory symptoms and posttraumatic stress disorder 5-6 years post-9/11 in responders enrolled in the World Trade Center Health Registry. Am J Ind Med. 2013;56(11):1251-61. https://doi.org/10.1002/ajim.22217.

20. Brackbill RM, Cone JE, Farfel MR, Stellman SD. Chronic physical health consequences of being injured during the terrorist attacks on world trade center on September 11, 2001. Am J Epidemiol. 2014;179(9):1076-85. https://doi. org/10.1093/aje/kwu022.

21. Brackbill RM, Thorpe LE, DiGrande L, Perrin M, Sapp JH 2nd, $\mathrm{Wu} \mathrm{D}$, et al. Surveillance for World Trade Center disaster health effects among survivors of collapsed and 
damaged buildings. Morb Mortal Wkly Rep Surveill Summ. 2006;55(2):1-18.

22. Weathers F, Litz BT, Herman DS, Huska JA, Keane TM. The PTSD checklist (PCL): reliability, validity, and diagnostic utility. San Antonio, TX: Annual Convention of the International Society for Traumatic Stress Studies; 1993.

23. American Psychiatric Association. Diagnostic and statistical manual of mental disorders, (DSM-IV). Arlington, VA: American Psychiatric Publishing; 1994.

24. Blanchard EB, Jones-Alexander J, Buckley TC, Forneris CA. Psychometric properties of the PTSD checklist (PCL). Behav Res Ther. 1996;8:669-73.

25. Whealin JM, Stotzer R, Nelson D, Li F, Liu-Tom HT, Pietrzak RH. Evaluating PTSD prevalence and resilience factors in a predominantly Asian American and Pacific Islander sample of Iraq and Afghanistan Veterans. $J$ Affect Disord. 2013;150(3):1062-8. https://doi.org/10.1016/j. jad.2013.05.044.

26. Kessler RC, Andrews G, Colpe LJ, et al. Short screening scales to monitor population prevalences and trends in nonspecific psychological distress. Psychol Med. 2002;32(6): 959-76. https://doi.org/10.1017/S0033291702006074.

27. Sorkin DH, Ngo-Metzger Q, Nguyen H. Assessing the mental health needs and barriers to care among a diverse sample of Asian American older adults. J Gen Intern Med. 2011;26(6):595-602. https://doi.org/10.1007/s11606-0101612-6.

28. Kessler RC, Barker PR, Colpe LJ, Epstein JF, Gfroerer JC, Hiripi E, et al. Screening for serious mental illness in the general population. Arch Gen Psychiatry. 2003;60(2):184-9. https://doi.org/10.1001/archpsyc.60.2.184.

29. Brackbill RM, Hadler JL, DiGrande L, Ekenga CC, Farfel $\mathrm{MR}$, Friedman S, et al. Asthma and posttraumatic stress symptoms 5 to 6 years following exposure to the World Trade Center terrorist attack. JAMA. 2009;302(5):502-16. https://doi.org/10.1001/jama.2009.1121.

30. Hochberg Y. A sharper Bonferroni procedure for multiple tests of significance. Biometrika. 1988;75(4):800-2. https://doi.org/10.1093/biomet/75.4.800.

31. Hochberg Y, Benjamini Y. More powerful procedures for multiple significance testing. Stat Med. 1990;9(7):811-8. https://doi.org/10.1002/sim.4780090710.

32. SAS Institute Inc. USA, SAS For Windows 8 Release. 2014.

33. De Bocanegra HT, Brickman E. Mental health impact of the World Trade Center attacks on displaced Chinese workers. $J$ Trauma Stress. 2004;17(1):55-62. https://doi.org/10.1023 /B:JOTS.0000014677.20261.dd.

34. De Bocanegra HT, Moskalenko S, Kramer EJ. PTSD, depression, prescription drug use, and health care utilization of Chinese workers affected by the WTC attacks. $J$ Immigr Minor Health. 2006;8(3):203-10. https://doi.org/10.1007 /s10903-006-9323-0.

35. Digrande L, Neria Y, Galea S, Brackbill RM, Pulliam P. Long-term posttraumatic stress symptoms among 3,271 civilian survivors of the september 11, 2001, terrorist attacks on the World Trade Center. Am J Epidemiol. 2011;173(3): 271-81. https://doi.org/10.1093/aje/kwq372.

36. Perrin MA, DiGrande L, Wheeler K, Thorpe L, Farfel M, Brackbill R. Differences in PTSD prevalence and associated risk factors among World Trade Center disaster rescue and recovery workers. Am J Psychiatr. 2007;164(9):1385-94. https://doi.org/10.1176/appi.ajp.2007.06101645.

37. Bowler RM, Han H, Gocheva V, Nakagawa S, Alper H, DiGrande L, et al. Gender differences in probable posttraumatic stress disorder among police responders to the 2001 World Trade Center terrorist attack. Am J Ind Med. 2010;53(12):1186-96. https://doi.org/10.1002/ajim.20876.

38. Galea S, Ahern J, Resnick H, Kilpatrick D, Bucuvalas M, Gold J, et al. Psychological sequelae of the September 11 terrorist attacks in New York City. $N$ Engl J Med. 2002;346(13):982-7. https://doi.org/10.1056 /NEJMsa013404.

39. Chiu KB, de Roon-Cassini TA, Brasel KJ. Factors identifying risk for psychological distress in the civilian trauma population. Acad Emerg Med. 2011;18(11):1156-60. https://doi.org/10.1111/j.1553-2712.2011.01206.x.

40. Parto JA, Evans MK, Zonderman AB. Symptoms of posttraumatic stress disorder among urban residents. J Nerv Ment Dis. 2011;199(7):436-9. https://doi.org/10.1097 /NMD.0b013e3182214154.

41. Kim C, Sakamoto A. Have Asian American men achieved labor market parity with White men? Am Sociol Rev. 2010;75(6):934-57. https://doi.org/10.1177 $/ 0003122410388501$.

42. Zeng Z, Xie Y. Asian Americans' earnings disadvantage reexamined: the role of place of education. Am J Sociol. 2004;190(5):1075-108.

43. Uba L. Asian Americans: personality patterns, identity, and mental health. New York: Guilford Press; 2003.

44. Shiratori Y, Samuelson KW. Relationship between posttraumatic stress disorder and asthma among New York area residents exposed to the World Trade Center disaster. $J$ Psychosom Res. 2012;73(2):122-5. https://doi.org/10.1016 /j.jpsychores.2012.05.003.

45. Hinton DE, Pich V, Marques L, Nickerson A, Pollack MH. Khyâl attacks: a key idiom of distress among traumatized Cambodia refugees. Culture Med Psychiatry. 2010;34(2): 244-78. https://doi.org/10.1007/s11013-010-9174-y.

46. Chen H, Kramer EJ, Chen T. The bridge program: a model for reaching Asian Americans. Psychiatr Serv. 2003;54(10): 1411-2.

47. U.S. Census Bureau. 2010-2014 American Community Survey 5-Year Estimates. http://factfinder.census. gov/faces/nav/jsf/pages/index.xhtml. Accessed July 4, 2016.

48. Ryan C. Language use in the United States: 2011. American Community Survey Reports. 2013;22:1-16. 